\title{
Improved adequacy of endometrial tissue sampled from postmenopausal women using the MyoSure Lite hysteroscopic tissue removal system versus conventional curettage
}

\author{
This article was published in the following Dove Press journal: \\ International Journal of Women's Health \\ 27 October 2017 \\ Number of times this article has been viewed
}

\author{
Peter Rosenblatt' \\ Sara Barcia ${ }^{2}$ \\ Anthony DiSciullo' \\ Hussein Warda' \\ 'Division of Urogynecology, Mount \\ Auburn Hospital, Harvard Medical \\ School, Cambridge, MA, 'Department \\ of Pathology, Mount Auburn Hospital, \\ Harvard Medical School, Cambridge, \\ MA, USA
}

\begin{abstract}
Objective: To compare the diagnostic suitability of endometrial tissues obtained from postmenopausal women using the MyoSure Lite tissue removal system versus conventional curettage.

Materials and methods: Endometrial tissue was sampled in hysteroscopically normal extirpated uteri from seven postmenopausal women (65.9 \pm 6.6 years old) using both hysteroscopicguided morcellation and curettage (two quadrants/uterus with each method). Endometrial sampling was performed immediately after hysterectomy for benign reasons unrelated to uterine pathology. Retrieved endometrial tissue samples were evaluated for volume and diagnostic suitability by a pathologist who was masked to the sampling technique used.

Results: Endometrial tissue sampling times were similar for morcellation ( $44 \pm 23 \mathrm{~s})$ and curettage (47 $\pm 38 \mathrm{~s})$. Mean tissue volume retrieved with MyoSure $\left(1,411 \pm 775 \mathrm{~mm}^{3}\right)$ was significantly greater than with curettage $\left(1 \pm 2 \mathrm{~mm}^{3} ; p=0.0004\right.$, two-tailed $t$-test), with larger intact tissue fragments retrieved with morcellation. Both specimen volume and quality obtained by MyoSure Lite were deemed to be significantly better for histologic assessment than the tissues obtained with curettage ( $p=0.0006$ by Fisher's exact test and $p=0.0137$ by chi-square test, respectively). With dilation and curettage, samples were frequently too scanty for evaluation. Diagnostic concurrence between MyoSure Lite/dilation and curettage samples and histopathology of full-thickness samples taken afterward was also significantly better with MyoSure Lite than with curettage $(p=0.0210)$.

Conclusion: Endometrial tissue sampling using the minimally invasive MyoSure Lite hysteroscopic tissue removal system may provide larger volumes of higher-quality endometrial tissue specimens for pathology assessment compared to specimens obtained using conventional curettage, in postmenopausal women.
\end{abstract}

Keywords: hysteroscopic morcellation, curettage, endometrial tissue sampling, endometrial pathology, abnormal uterine bleeding, extirpated uterus, postmenopausal

\section{Introduction}

Unexplained abnormal uterine bleeding occurs in up to $10 \%$ of women over 55 years old, and suspicion of endometrial pathologies including leiomyomas, polyps, and carcinoma all require thorough diagnostic evaluation. ${ }^{1-5}$ Postmenopausal women are at risk for all of these uterine abnormalities. Endometrial carcinoma now supersedes invasive cervical cancer as the most common gynecologic malignancy in the USA ${ }^{3}$ and other global regions. ${ }^{4}$ More than $90 \%$ of cases occur in women older than 50 years, with the mean age at diagnosis in the USA being 62 years. ${ }^{5,6}$ Abnormal bleeding
Correspondence: Peter Rosenblatt Boston Urogynecology Associates, 725 Concord Ave., Suite 1200,

Cambridge, MA 02138, USA

Tel +l 6173545452

Fax +I 6173540458

Email prosenbl@mah.harvard.edu 
accompanies $90 \%$ of endometrial cancers. ${ }^{7}$ Whereas most causes of postmenopausal bleeding are benign, a significant proportion of these patients, indeed, have malignancy. ${ }^{5-7}$ Therefore, any atypical bleeding in postmenopausal women needs to be thoroughly investigated to rule out carcinoma. It is essential to have reliable methods to definitively assess endometrial abnormalities for providing optimal gynecologic health care. ${ }^{6}$ Although uterine imaging by transvaginal ultrasound, saline infusion sonohysterography, and diagnostic hysteroscopy provide important anatomic information, ${ }^{8}$ histologic evaluation of endometrial tissue samples remains the definitive approach for assessing endometrial pathology. ${ }^{9}$ However, current endometrial sampling methods have important limitations that need to be overcome.

Dilation and curettage (D\&C) has long been a mainstay approach for obtaining uterine lining samples for histologic analysis. Diverse endometrial sampling technologies have been developed to supplant D\&C, including aspiration (eg, Vabra; Berkeley Medevices, Inc., Richmond, CA, USA), suction biopsy (eg, Pipelle; Pipelle de Cornier, Paris, France), and excisional biopsy with or without hysteroscopic guidance, each having their own unique benefits, limitations, and risks. ${ }^{9}$ Important considerations when selecting an endometrial sampling technique include sensitivity and specificity for capturing global versus focal lesions, risk of serious adverse events, ease of operation, cervical dilation requirements, patient comfort and satisfaction levels, and instrumentation and procedural costs. An enduring problem is that the commonly employed endometrial sampling approaches often yield poor-quality tissue samples that are inadequate for histologic diagnosis, and insufficient sampling is more than twice as common with older (ie, $\geq 60$ years old) versus younger women. ${ }^{10}$

Hysteroscopic morcellating tissue removal devices have emerged as a new tool for concomitantly visualizing and resecting focal intrauterine lesions, and have advantages over previous resectoscopic techniques by effectively removing endometrial pathologies while reducing the procedural time and adverse event rates. ${ }^{1-14}$ This study compares the suitability of the MyoSure Lite Tissue Removal System (Hologic Inc., Marlborough, MA, USA) to conventional sharp curettage for collecting high-quality endometrial tissue samples sufficient for reliable histologic assessment.

\section{Materials and methods}

\section{Study design and patients}

This was a prospective pilot study that compared two endometrial sampling procedures. We evaluated endometrial tissue adequacy for diagnostic analysis after sampling by hysteroscopic morcellation versus conventional curettage, from the uteri of postmenopausal women undergoing hysterectomy for benign causes. The study was performed between July 20, 2015 and June 29, 2016. All tissue donors satisfied all the study criteria (detailed below) and provided written informed consent before enrollment. The study protocol was approved by and performed with oversight of the Institutional Review Board at Mount Auburn Hospital (Cambridge, MA, USA). Tissue donors were de-identified in all study documents, and no study procedures were performed on living humans. This investigation adhered to the tenets of the Declaration of Helsinki and was Health Insurance Portability and Accountability Act compliant.

\section{Inclusion criteria}

Primary enrollment criteria included: 1) women aged 50-75 years undergoing hysterectomy for benign reasons unrelated to endometrial abnormalities, for example, pelvic organ prolapse; 2) postmenopausal status with absence of menses $>1$ year; and 3 ) extirpated uterus that was hysteroscopically normal.

\section{MyoSure device}

The MyoSure Lite Hysteroscopic Tissue Removal System (Hologic Inc.) is a US Food and Drug Administrationapproved (K091100) device for resecting and removing intrauterine tissue including polyps $\leq 3 \mathrm{~cm}$ in diameter. ${ }^{15}$ The hand-held hysteroscopic system houses a hysteroscope and a disposable morcellator within a hollow stainless steel tube with $6.25 \mathrm{~mm}$ outer diameter. The mechanical drive assembly connects to a shaft of $3 \mathrm{~mm}$ diameter that drives the cutting blade. Under hysteroscopic guidance, the cutting blade contacts the target tissue through a side-facing $10.2 \mathrm{~mm}$ long and $1.5 \mathrm{~mm}$ deep channel on the morcellator distal shaft. Upon motor activation, the blade guard covering the window retracts, and the hardened stainless steel blade engages its dual cutting motion, rotating at 8,075 rpm while oscillating at 3 cycles/s. The MyoSure Lite system is compatible with typical fluid control systems. Physiological saline is used as the distending medium, and vacuum action retrieves the excised tissue through the shaft via the cutting port. The Lite device has a rated tissue removal rate of $7.0 \mathrm{~g} / \mathrm{min}$ (polyps) at $300 \mathrm{mmHg}$ suction pressure and $100 \mathrm{mmHg}$ intrauterine pressure. ${ }^{16}$

\section{Tissue sampling}

Donor uteri were collected in the operating room, de-identified after confirming study eligibility, transported to the pathology 
laboratory, and sampled within 4 hours ex vivo. The uteri were secured to a dissecting/examination tray with the anterior wall downward; the fallopian tubes were clamped, and the cervix was stabilized using two tenaculums and the residual cervical length measured to assure adequate length for performing hysteroscopy. After cervical dilation to $\leq 6 \mathrm{~mm}$, hysteroscopy was performed to evaluate the cavity for general appearance and presence of focal lesions (exclusionary, except for minor scarring/adhesions), with the findings archived by digital image acquisition. For curettage, two cavity walls (anterior and left lateral) were sampled by a gynecologic surgeon (HW or PR) using a \#1 sharp curette, with both pre- and post-collection images taken hysteroscopically. The uterus was then inverted $180^{\circ}$ along the axial plane, and therefore, the posterior wall was downward. Tissue sampling of the posterior and right lateral intrauterine walls was performed using the MyoSure Lite system, inserted through the hysteroscope, using an Aquilex fluid management system (Hologic Inc.) with suction set to medium and intrauterine pressure set at $80 \mathrm{mmHg}$. Intrauterine images were taken again after hysteroscopic morcellation.

\section{Pathology assessment}

Tissues obtained by curettage and hysteroscopic morcellation were submitted to the pathology department for blinded gross and histologic assessment, the latter performed by microscopic evaluation of hematoxylin and eosin-stained sections of formalin-fixed, paraffin-embedded tissue blocks by a single observer (SB). Full-thickness samples of the anterior and posterior uterine walls were then collected and used for comparison to the experimental biopsy samples.

\section{Outcome measures}

Study outcomes included sampling time, total sample volume retrieved, maximum intact tissue fragment size, pathologist assessment of tissue suitability for histologic analysis, and microscopic description.

\section{Statistical analyses}

Categorical variables were compared using either Fisher's exact test (with only two possible outcomes) or chi-square assessment (with $>2$ possible outcomes), as appropriate and indicated. Continuous variables were compared by Student's $t$-test, paired when appropriate. For all statistical tests, two-tailed $p$-values $<0.05$ indicated statistically significant differences. Data are presented as mean \pm SD or as $n(\%)$ of cohort. Data were analyzed using Excel 2010 (Microsoft
Corp., Redmond, WA, USA) and Prism v.5.03 (GraphPad Software Inc., San Diego, CA, USA).

\section{Results}

We obtained specimens from seven women aged 65.9 \pm 6.6 years (range 58-74 years), all of whom underwent a hysterectomy for uterine and/or uterovaginal prolapse. Four study uteri were sampled by one surgeon (PR) and three were sampled by another surgeon (HW). Three additional donor uteri were excluded from evaluation when post-extirpation hysteroscopy revealed focal lesions. Mean remnant cervical length in extirpated uteri was $1.3 \mathrm{~cm}$ (range $0.5-4.0 \mathrm{~cm}$ ), which was deemed sufficient for stable tenaculum attachment during examination and tissue sampling (Table 1). Average cutting times for sample retrieval were similar with MyoSure Lite (44 $\pm 23 s)$ and curettage ( $47 \pm 38 s ; p=0.8466)$, with the longest cutting time for hysteroscopic morcellation being $80 \mathrm{~s}$ and for curettage being $120 \mathrm{~s}$. Curettage employed a mean 14.3 \pm 7.3 instrument passes for sample collection. Mean tissue volume retrieved with MyoSure Lite $\left(1,411 \pm 775 \mathrm{~mm}^{3}\right)$ was significantly greater than with curettage $\left(1 \pm 2 \mathrm{~mm}^{3} ; p=0.0004\right.$, paired $t$-test; Table 2$)$, with larger intact tissue fragments retrieved with morcellation. Both specimen volume and quality obtained by MyoSure Lite were deemed to be significantly better for histologic assessment than the tissues obtained with curettage ( $p=0.0006$ by Fisher's exact test and $p=0.0137$ by chi-square test, respectively). Diagnostic concurrence between MyoSure Lite/D\&C samples and histopathology of full-thickness samples taken afterward was also significantly better with MyoSure Lite than with curettage $(p=0.0210)$.

Table I Baseline characteristics of donors and donor uteri

\begin{tabular}{ll}
\hline Parameter & Value (n=7) \\
\hline Subject parameters & \\
Age, years & \\
$\quad$ Mean \pm SD & $65.9 \pm 6.6$ \\
$\quad$ Range & $58-74$ \\
Reason for hysterectomy, $n(\%)$ & \\
$\quad$ Prolapse, nonspecified & $4(57)$ \\
Prolapse, uterine & $2(29)$ \\
$\quad$ Prolapse, vaginal & $1(14)$ \\
Uterus gross characteristics & \\
Cervix remaining, cm & \\
$\quad$ Mean \pm SD & $1.3 \pm 1.2$ \\
$\quad$ Range & $0.5-4.0$ \\
Endometrial appearance at hysteroscopy, $\mathrm{n}(\%)$ & \\
$\quad$ Atrophic & $7(100)$ \\
Leiomyoma & $0(0)$ \\
Polyp & $0(0)$ \\
Normal/functional & $0(0)$ \\
Other abnormality & $0(0)$ \\
\hline
\end{tabular}


Table 2 Tissue sampling and pathology observations

\begin{tabular}{|c|c|c|c|}
\hline \multirow{3}{*}{$\begin{array}{l}\text { Parameter } \\
\text { Tissue sampling } \\
\text { parameters }\end{array}$} & \multicolumn{2}{|c|}{$\begin{array}{l}\text { Value ( } n=7 \text { for each } \\
\text { method) }\end{array}$} & \multirow[t]{3}{*}{$p$-value } \\
\hline & MyoSure & Curette & \\
\hline & \multicolumn{2}{|l|}{ Lite } & \\
\hline \multicolumn{4}{|l|}{ MyoSure cutting time, $s$} \\
\hline Mean \pm SD & $44 \pm 13$ & $47 \pm 38$ & \multirow[t]{2}{*}{0.8466} \\
\hline Range & $21-80$ & $14-120$ & \\
\hline \multicolumn{4}{|l|}{ Pathology findings } \\
\hline \multicolumn{4}{|l|}{ Sample volume, $\mathrm{mm}^{3}$} \\
\hline Mean \pm SD & $\mathrm{I}, 4 \mathrm{I} \mathrm{I} \pm 775$ & $I \pm 2$ & \multirow[t]{2}{*}{0.0004} \\
\hline Range & $760-2,970$ & $0-6$ & \\
\hline Fragment size, largest, $\mathrm{mm}$ & $5 \pm 1$ & $I \pm I$ & $<0.000$ I \\
\hline \multicolumn{4}{|c|}{ Specimen volume adequacy, n (\%) } \\
\hline Satisfactory & $7(100)$ & $0(0)$ & \multirow[t]{2}{*}{0.0006} \\
\hline Limited & $0(0)$ & $7(100)$ & \\
\hline \multicolumn{4}{|c|}{ Specimen quality assessment, $\mathrm{n}(\%)$} \\
\hline Very satisfied & $4(57)$ & $0(0)$ & \multirow[t]{4}{*}{0.0137} \\
\hline Satisfied & $2(29)$ & $0(0)$ & \\
\hline Unsatisfied & $0(0)$ & $2(29)$ & \\
\hline Very unsatisfied & $I(14)$ & $5(7 I)$ & \\
\hline \multicolumn{4}{|l|}{ Tissues present, $\mathrm{n}(\%)$} \\
\hline Endometrium only & $0(0)$ & $0(0)$ & \multirow[t]{4}{*}{$0.1923^{\mathrm{a}}$} \\
\hline Endometrium and stroma & $7(100)$ & $2(29)$ & \\
\hline Myometrium & $2(29)$ & $2(29)$ & \\
\hline No tissue & $0(0)$ & $3(43)$ & \\
\hline \multicolumn{4}{|l|}{ Glands:stroma area ratio, n (\%) } \\
\hline Glands $>$ stroma & $0(0)$ & $0(0)$ & \multirow[t]{4}{*}{$0.0699^{b}$} \\
\hline Glands = stroma & $0(0)$ & $0(0)$ & \\
\hline Glands $<$ stroma & $7(100)$ & $3(43)$ & \\
\hline Indeterminate & $0(0)$ & $4(57)$ & \\
\hline \multicolumn{4}{|l|}{ Diagnostic concurrence, $\mathrm{n}(\%)^{\mathrm{c}}$} \\
\hline Yes & $7(100)$ & $2(29)$ & \multirow[t]{2}{*}{0.0210} \\
\hline No/inconclusive & $0(0)$ & $5(7 I)$ & \\
\hline
\end{tabular}

Notes: Continuous variables were compared by two-tailed $t$-test. Categorical variables were compared using Fisher's exact test (two possible outcomes) or chi-square analysis (specimen quality assessment). a ${ }^{2}$ utcomes were segregated by "no tissue" versus any tissue present, and compared using Fisher's exact test. bOutcomes were segregated by "indeterminate" versus any possible assessment, and compared using Fisher's exact test. 'All extirpated uteri were diagnosed as atrophic and containing no focal adhesions by histologic evaluation of full-thickness excisional tissue samples taken after performing MyoSure Lite/curette sampling.

\section{Discussion}

Uterine pathologies including hyperplasia, leiomyomas, polyps, and carcinoma are responsible for significant morbidity in women across the globe. ${ }^{1-7}$ Hyperplasia, atrophy, polyps, leiomyosarcomas, and, most critically, carcinoma, all increase in frequency after menopause. ${ }^{1-7}$ The cumulative incidence of leiomyomas is highest in women approaching 50 years of age and continues to increase in postmenopausal women, albeit at a slower rate. ${ }^{1}$ In most instances, histologic assessment of endometrial tissue samples remains the best approach for assessing uterine lining abnormalities and planning the next clinical course of action. Global endometrial sampling is commonly performed using $\mathrm{D} \& \mathrm{C}$, although with highly variable outcomes in terms of specimen volume and suitability for pathological accuracy, procedural ease, cost and duration, and patient comfort. ${ }^{10,11}$ For example, endometrial curettage evaluates more than half of the uterine cavity in fewer than $60 \%$ of D\&C procedures,${ }^{17}$ increasing the likelihood of false-negative determinations for focal lesions. Furthermore, total endometrial curettage may be insufficient for differentiating endometrial hyperplasia from carcinoma. ${ }^{18,19}$ A study on D\&C regarding biopsy specimen adequacy highlighted the common problem of insufficient endometrial tissue quantity and quality with increasing subject age. Inadequate specimens were obtained in 3\% of women aged $<40$ years, $6 \%$ of women aged $40-59$ years, and $15 \%$ of women aged $\geq 60$ years. ${ }^{10}$ A 2016 systematic review and meta-analysis of 12 studies involving 1,029 postmenopausal women with abnormal uterine bleeding indicated that, using hysteroscopy as a reference, the detection sensitivity of D\&C in endometrial cancer (90\%, range 50\%-100\%), atypical hyperplasia ( $82 \%$, range $56 \%-94 \%$ ), and endometrial disease including benign polyps (39\%, range $21 \%-69 \%$ ) is lower than previously believed. ${ }^{20}$ Additionally, the failure rate of endometrial sampling was $11 \%$ (range $1 \%-53 \%$ ), while insufficient samples occurred in 31\% (range 7\%-76\%) of postmenopausal women studied. We evaluated the MyoSure Lite small-bore hysteroscopic tissue morcellator for its ability to collect suitable volumes of high-quality endometrial samples in a human extirpated uterus model.

The primary study finding is that endometrial sampling with the MyoSure Lite system consistently retrieved an ample quantity of sufficiently intact target tissue for accurate and detailed histologic evaluation, compared to the samples obtained by D\&C. Both tissue volume and maximum recovered fragment size in samples obtained with MyoSure Lite were significantly larger than in curettage samples from the same uteri. The masked pathologist rated all MyoSure Litederived samples to be adequate for analyses, whereas all curettage samples were deemed to be of limited adequacy, primarily due to insufficient specimen size. There were no notations of tissue fragmentation or crush artifact occurrences with either sampling method. There was obvious dichotomy in terms of pathologist satisfaction with the overall sample quality, whereby all curettage samples earned an "unsatisfied" (29\%) or "very unsatisfied" (71\%) ranking. Only a single MyoSure Lite specimen (14\%) fell into the unsatisfied spectrum. All postmenopausal uteri in this study had atrophic endometrium; this was identified in $100 \%$ of MyoSure Lite samples, but only $29 \%$ of the curettage samples. Thus, in our hands, the hysteroscopic morcellation system outperformed 
$\mathrm{D} \& \mathrm{C}$ in procuring endometrial samples of sufficient quality for histologic analysis. When compared to the full-thickness sections of corresponding uteri, the morcellation biopsies were determined to be better representatives than the D\&C samples.

The MyoSure Lite device provides technical advantages over D\&C. While tissue collection times were similar with both methods, the morcellator is guided by direct visualization whereas D\&C remains a blind technique. The uterine lining is continually imaged with the MyoSure Lite hysteroscope, allowing real-time assessment of the intrauterine workspace to ensure thorough endometrial sampling. Unlike D\&C, repeated instrument insertions are not needed with the MyoSure Lite, rendering it a much less-invasive sampling. The blunt distal end of the morcellator has larger surface area compared to standard sharp curettes, which theoretically lowers the likelihood of inadvertent uterine puncture. Indeed, the MyoSure Lite system has a large and increasing body of favorable safety data. ${ }^{12,14,21}$ Whereas endometrial sampling by D\&C is an open system for tissue collection, the MyoSure Lite morcellator is part of a closed-loop, continuously irrigated tissue extraction system, wherein the resected endometrial tissue specimen is immediately suctioned into the instrument and specimen fragments are immobilized in an external collection trap, thereby precluding unintentional sample loss. Thus, the MyoSure Lite system captures essentially all endometrial tissues removed by the high-speed blade, resulting in samples that are wellsuited for histologic analysis.

Study strengths included a design that directly allowed comparison of MyoSure Lite to D\&C sampling in the same uterus and the masking of the interpreting pathologist. Primary limitations of this pilot study included the relatively small number of uteri tested and the ex vivo nature of our experimental model. Additionally, no uterine pathology other than atrophic endometrium was included in the study analysis, so further studies are needed to determine if MyoSure Lite sampling identifies more pathology than traditional D\&C. This will be explored in planned prospective randomized studies of endometrial sampling in human volunteers. The MyoSure Lite hysteroscopic tissue removal system may provide larger amounts of higher-quality endometrial tissue specimens than conventional D\&C.

\section{Acknowledgments}

The authors thank Matt Silverman PhD (Biomedical Publishing Solutions, Delray Beach, FL, USA) for analytical, statistical, and editing services in preparing this manuscript. This study was funded by Hologic Inc., the study device manufacturer. This work was presented, in part, in poster format at the 2015 Annual Congress of the American Association of Gynecologic Laparoscopists, November 15-19, Las Vegas, NV, USA.

\section{Author contributions}

PR designed the study and recruited participants. All authors were involved in data acquisition and interpretation, drafting/ revising the manuscript, and critical review, and approved the submission of the report for publication.

\section{Disclosure}

Dr Rosenblatt received speaker fees from Hologic. The authors report no other conflicts of interest in this work.

\section{References}

1. Khan AT, Shehmar M, Gupta JK. Uterine fibroids: current perspectives. Int $J$ Womens Health. 2014;6:95-114.

2. Dreisler E, Stampe Sorensen S, Ibsen PH, Lose G. Prevalence of endometrial polyps and abnormal uterine bleeding in a Danish population aged 20-74 years. Ultrasound Obstet Gynecol. 2009;33(1):102-108.

3. Siegel RL, Miller KD, Jemal A. Cancer statistics, 2016. CA Cancer J Clin. 2016;66(1):7-30.

4. Ferlay J, Soerjomataram I, Dikshit R, et al. Cancer incidence and mortality worldwide: sources, methods and major patterns in GLOBOCAN 2012. Int J Cancer. 2015;136(5):E359-E386.

5. National Cancer Institute. U.S. Department of Health and Human Services. Surveillance, Epidemiology, and End Results Program Statistics Fact Sheet: Endometrial Cancer. Available from: http://seer. cancer.gov/statfacts/html/corp.html. Accessed April 12, 2017.

6. American College of Obstetricians and Gynecologists. Practice Bulletin No. 149: Endometrial cancer. Obstet Gynecol. 2015;125(4): 1006-1026.

7. Sorosky JI. Endometrial cancer. Obstet Gynecol. 2012;120:383-397.

8. Epstein E, Ramirez A, Skoog L, Valentin L. Transvaginal sonography, saline contrast sonohysterography and hysteroscopy for the investigation of women with postmenopausal bleeding and endometrium $>5 \mathrm{~mm}$. Ultrasound Obstet Gynecol. 2001;18(1):157-162.

9. Du J, Li Y, Lv S, et al. Endometrial sampling devices for early diagnosis of endometrial lesions. J Cancer Res Clin Oncol. 2016;142(12): 2515-2522.

10. Kandil D, Yang X, Stockl T, Liu Y. Clinical outcomes of patients with insufficient sample from endometrial biopsy or curettage. Int $J$ Gynecol Pathol. 2014;33(5):500-506.

11. Cohen S, Greenberg JA. Hysteroscopic morcellation for treating intrauterine pathology. Rev Obstet Gynecol. 2011;4(2):73-80.

12. Bhalani V, Chang, A, Adkins C, Chen SH, Scheiber MD. Fertility outcomes after hysteroscopic morcellation of intrauterine leiomyomas and polyps. J Reprod Med. 2016;61:327-335.

13. Scheiber M, Chen S. A prospective multi-center registry of patients undergoing hysteroscopic morcellation of uterine polyps and myomas. J Gynecol Surg. 2016;32(6):318-323.

14. Rubino RJ, Lukes AS. Twelve-month outcomes for patients undergoing hysteroscopic morcellation of uterine polyps and myomas in an office or ambulatory surgery setting. J Minim Invasive Gynecol. 2015;22(2):285-290.

15. MyoSure ${ }^{\mathrm{TM}}$ Hysteroscopic Tissue Removal System 510(k) summary. Framingham, MA: Interlace Medical; prepared March 30, 2009. Available from: http://www.accessdata.fda.gov/cdrh_docs/pdf9/K091100. pdf. Accessed April 12, 2017. 
16. Hologic Inc. Myosure tissue removal system: Physician brochure. Available from: http://www.hologic.com/downloads?field_product_category_new_value=intervention_and_treatment\&field_subcategory_ new_value=gynecologic_surgery\&title. Accessed April 13, 2017.

17. Dijkhuizen FP, Mol BW, Brölmann HA, Heintz AP. The accuracy of endometrial sampling in the diagnosis of patients with endometrial carcinoma and hyperplasia: a meta-analysis. Cancer. 2000;89(8): 1765-1772.

18. Demirkiran F, Yavuz E, Erenel H, Bese T, Arvas M, Sanioglu C. Which is the best technique for endometrial sampling? Aspiration (pipelle) versus dilatation and curettage (D\&C). Arch Gynecol Obstet. 2012; 286(5):1277-1282.
19. Bourdel N, Chauvet P, Tognazza E, Pereira B, Botchorishvili R, Canis M. Sampling in atypical endometrial hyperplasia: which method results in the lowest underestimation of endometrial cancer? A systematic review and meta-analysis. J Minim Invasive Gynecol. 2016;23(5): 692-701.

20. van Hanegem N, Prins MM, Bongers MY, et al. The accuracy of endometrial sampling in women with postmenopausal bleeding: a systematic review and meta-analysis. Eur J Obstet Gynecol Reprod Biol. 2016;197: $147-155$.

21. Haber K, Hawkins E, Levie M, Chudnoff S. Hysteroscopic morcellation: review of the manufacturer and user facility device experience (MAUDE) database. J Minim Invasive Gynecol. 2015;22(1):110-114.

\section{Publish your work in this journal}

The International Journal of Women's Health is an international, peerreviewed open-access journal publishing original research, reports, editorials, reviews and commentaries on all aspects of women's healthcare including gynecology, obstetrics, and breast cancer. The manuscript management system is completely online and includes a very quick and fair peer-review system, which is all easy to use. Visit http://www.dovepress.com/testimonials.php to read real quotes from published authors.

Submit your manuscript here: http://www.dovepress.com/international-journal-of-womens-health-journal 